IAC-06-B6.1.2

\title{
SURVEY AND CHASE: A NEW METHOD OF OBSERVATIONS FOR THE MICHIGAN ORBITAL DEBRIS SURVEY TELESCOPE (MODEST)
}

\author{
Kira J. Abercromby \\ ESCG/Jacobs, Houston, TX, USA \\ kira.abercromby-1@nasa.gov \\ Patrick Seitzer \\ University of Michigan, Ann Arbor, MI, USA \\ pseitzer@umich.edu \\ Heather M. Rodriguez \\ ESCG/Jacobs, Houston, TX, USA \\ heather.m.rodriguez@nasa.gov \\ Edwin S. Barker, Mark J. Matney \\ NASA Johnson Space Center, Houston, TX, USA \\ edwin.s.barker@nasa.gov, mark.matney-1@nasa.gov
}

\begin{abstract}
The Michigan Orbital DEbris Survey Telescope (MODEST) is normally used to survey the geosynchronous orbit (GEO) environment to obtain a statistical assessment of the debris population. Due to the short time that the object is in the field-of-view (usually five minutes), it is common practice to assume a circular orbit when calculating the orbit from this limited observational arc. Some objects in the GEO regime are GEO-transfer orbit (GTO) objects which are observed at their apogee or objects with varying eccentricities such as those with high area-to-mass ratios. For these objects, an assumed circular orbit (ACO) prediction would not be accurate. After MODEST was modernized in March 2005 and brought under computer control, it became possible to use the telescope in modes other than tracking at the sidereal rate. Three data runs were conducted to test the orbit determination program, the ability to transfer data effectively between site locations, and to determine if objects could be re-acquired with this method. We report in this paper our initial efforts to determine full orbits based on follow-up observations after the initial detection in survey mode. Our long-term goal is to construct a system which can detect and follow-up an object in any orbit at GEO. This paper reports our first steps towards that goal. During the three data collections, only circular orbit objects were obtained. Although this allowed for testing of the process, further tests must be conducted in an effort to target GTO and high area-to-mass objects.
\end{abstract}

\section{INTRODUCTION}

With the modernization of the Michigan Orbital DEbris Survey Telescope (MODEST) in March 2005, observations in modes other than tracking at the sidereal rate were possible. This allowed for efforts to commence determining the environment more accurately by obtaining accurate orbits on all types of objects. The best method through which one would determine an orbit of an object is to track it specifically.
However, in an effort to retain the survey capabilities of MODEST, a process was tested that allowed the retention of some survey data while doing follow-ups during parts of the evening.

These first efforts are based on determining orbits for geosynchronous orbit (GEO) objects which are in circular or near-circular orbits (with eccentricities less than 0.05). There are three reasons for doing so: 
- A GEO object that starts in a circular or low eccentric orbit will remain in such an orbit, unless it has a high area-to-mass (A/M) and is subject to significant solar radiation forces.

- Circular orbits are the simplest to predict and follow-up.

- There exists a large number of wellobserved, bright objects in the public United States Satellite Catalog at GEO which are on near-circular orbits and can serve as truth tests. How well do our derived orbits agree with the published ones?

Our long-term goal is to construct a system which can detect and follow-up an object in any orbit at GEO. This paper reports our first steps towards that goal.

The new method of observing entails using survey mode detections over a 5.3 minute timespan and propagating the orbits to predict specific right ascensions and declinations at future times. During the follow-up observations, longer time arcs are obtained and thus more accurate orbits are calculated. To date, survey and chase has been used on three observing runs.

Beginning in July 2005, MODEST surveyed for the first few hours of the night. Data was reduced in real-time at the telescope and sent to the National Aeronautics and Space Administration (NASA) Orbital Debris Program Office at Johnson Space Center (JSC) in Houston, Texas. Using assumed circular orbit (ACO) predictions, successful follow-up observations were conducted on those previously detected objects on the same night. With only an orbit propagator using a constrained eccentricity (referred to here as the constrained-ecc orbit propagator) available, two targets were recovered both with circular orbits.

Our next try was during the October 2005 run. The entire first night was observed in survey mode. Using an ACO prediction, MODEST was used in follow-up mode on the second and third night specifically targeting the fields where the projected orbits placed the objects. On night one, nine objects were detected, of which five were reacquired on the later nights. Two of the objects were circular uncorrelated targets (UCTs) and the other three were correlated targets (CTs) with circular orbits.

Finally, in January 2006, a "survey and chase" run was conducted similarly to the October 2005 run where the survey was completed on night one and the follow-up observations were conducted on subsequent nights. By acquiring a 24 hour arc of data, it was possible to use the non-constrained eccentricity orbit propagator (referred to here as the non-constrained-ecc orbit propagator) to calculate the orbit. Unfortunately, none of the objects recovered were UCTs.

Our results on the frequency of follow-up observations to determine an orbit from a short time arc survey observation agree in general with those reported earlier by the ESA debris group using the ESA Space Debris Telescope (SDT) on Tenerife ${ }^{1,2}$. One significant difference is that our initial observation period can be up to twice as long as obtained with the ESA SDT .

\section{MODEST}

Since early 2001, the University of Michigan and NASA have been using MODEST to observe orbital debris in the GEO environment. MODEST is a 0.6/0.9-m Schmidt telescope located at the Cerro Tololo Inter-American Observatory (CTIO) in Chile. A brief description of the system follows: for more details see References 3 and 4.

The telescope is equipped with a thinned $2048 \mathrm{x}$ 2048 pixel charge coupled device (CCD) with a field-of-view (FOV) of 1.3 degrees square and 2.318 arc-second pixels. In a 5 second exposure through a broad $\mathrm{R}$ filter a limiting magnitude of $\mathrm{R}$ $=18^{\text {th }}$ is reached with a signal to noise $(\mathrm{S} / \mathrm{N})$ of 10 .

In survey mode, the telescope tracks at the sidereal rate a fixed right ascension (RA) and declination (DEC) point close to the anti-solar point and outside of Earth shadow. During the exposure the charge on the CCD is shifted backwards such that GEO objects appear as point sources or short streaks, and stars appear drift as fixed length streaks. In the 5.3 minutes 

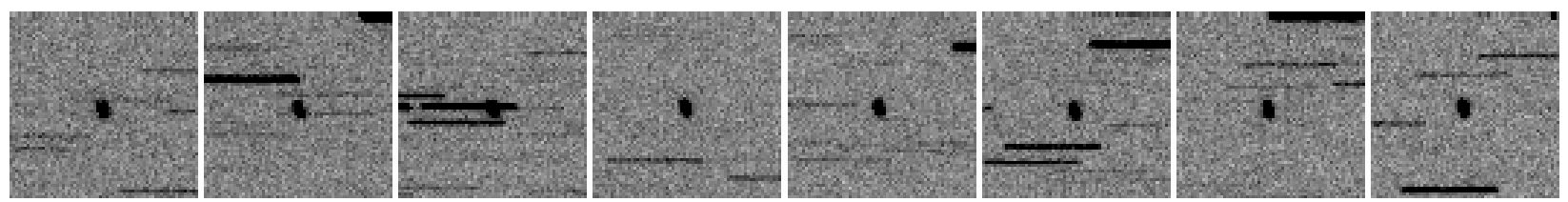

Fig. 1: An example of a survey detection sequence from MODEST. Each sub-image is 2.5 arc-minutes square. The GEO object is at the center of each sub-image, while the horizontal streaks are stars.

it takes a station-keeping GEO object to across the FOV, up to 8 independent positions can be measured. A minimum of 4 detections are required in this 5.3 minute window for a real detection. All correlated objects are visually examined to guard against false detections.

A circular orbit works well for most GEO objects, but for objects such as those in a GEO-transfer orbit (GTO) or with high $\mathrm{A} / \mathrm{M}$ ratios this process is not ideal. By using follow-up observations, either later in the same night or the next night, a longer time arc can be acquired such that a more accurate orbit is obtained. Figure 1 shows a sample detection sequence of a GEO object.

The system is designed to detect objects with angular rates between $+/-2$ arc-seconds/second in hour angle (HA) and +/- 5 arc-second/seconds in declination.

Data is reduced in real-time at the telescope and time stamped positions of survey objects are sent to NASA JSC in Houston, Texas, where the orbit and predictions for future location are calculated.

With such a short-arc observation (5.3 minutes out of a total orbital period of 1440 minutes), determination of a full parameter orbital solution is extremely uncertain, and hence the need for follow-up (chase) observations to determine the orbit accurately. The standard technique is to calculate a constrained-ecc (or circular) orbit based on the first 5.3 minutes of data.

\section{ORBIT PREDICTION}

The orbit prediction process determines the RA and DEC of the object at a specific time beginning 30 minutes after initial detection or anytime thereafter. However, depending on the orbit of the object, the likelihood of finding the object increases if the first follow-up observations are conducted within one hour of the initial observation. The fields where the object is predicted to be are calculated for every minute up to either four or eight hours after the initial detection. Following the results of the second test run in October 2005, the user has an option to determine the orbit using a circular orbit assumption (fixing the eccentricity) or to allow the eccentricity to be determined. As mentioned previously, if the actual orbit is eccentric the time arc of five minutes may not be sufficient to determine the orbit accurately using ACO.

The initial orbital parameters are calculated using initial radius and velocity vectors in equatorial coordinates (Earth-centered inertial) based on a method shown in detail in Reference 5. The program uses 100 Monte Carlo simulations to determine the best orbit, and ten possible orbits are sent to the prediction file. For each orbit a residual is calculated, which is a goodness of fit of the orbit to the detections. The fields where the object is predicted to be are computed by using the orbit with the smallest residual. These predicted fields are listed for every minute over the user specified time period. Observations can be added to the original set of detections to obtain a new prediction field for later in the same night or another night.

While calculating the orbit, the program determines whether or not the object is in shadow at the given time step. If it is in shadow, those fields are marked with the words "In Shadow" and are not sent in the prediction files to the telescope.

In these data sets presented in this paper, no correlation of the object to the catalogue is done while the observations are being conducted. To 
validate the prediction process, correlation with the United States Satellite Catalogue was conducted after the observing run was completed.

\section{CHASE MODE}

Predictions of where objects will be are sent from Houston to the telescope in Chile as quickly as possible. Turn-around time from receipt of initial survey positions in Houston to receipt of predicted future positions at the telescope was generally less than 1 hour.

Our initial experiments in follow-up (or chase mode) used the same observing technique as survey mode: the telescope tracked a predicted field at sidereal rate and the CCD was drift scanned backwards to remove the effect of the tracking. This allowed us to use the same observing and reduction software as survey mode, at the cost of observing time.

Given a list of an object's predicted position and time, the telescope was set at the position approximately 7 minutes ahead of the current starting time. Thus the object would drift across the center of the field about 10 minutes after the sequence started. This technique is largely insensitive to both timing and along-track errors, making it an excellent choice for our first experiments. Future observations will have the telescope come up on the object's predicted position, tracking at the predicted rates.

As per survey observations, a minimum of four measured positions were required on these chase observations for an object to be considered a real detection.

\section{RESULTS}

\section{July 2005}

The July 2005 trial was conducted within a night and was the first test to see if the turn around time of the observations was short enough to reacquire objects. The first two hours of each night were used for normal survey mode and follow-up objects were determined from those observations. On day of year (DOY) 189, three objects were detected and follow-up measurements were conducted two times within that same night. After the observing was completed for the night, a correlation with the catalogue found all three of the objects detected were correlated objects and were in circular orbits.

The second night in this test run (DOY 192) found six objects for follow-up. Four of the six objects were found on the first follow-up of the evening and three of those were found on a second follow-up attempt. The three found on both follow-ups were all CTs and were in circular orbits; however, the one that could not be found on the second attempt was a UCT.

The third night (DOY 193) of the July 2005 run, we found four objects and all four were reacquired two times during that night. Three of the objects were circular orbit CTs while one of the objects was a circular orbit UCT.

The July run proved that with a fast turn of less than 30 minutes, GEO objects can be reacquired with a predicted orbit. Since all of the objects found were in circular orbits, the time from the last observation to the first follow-up observations needs to be quicker to find eccentric orbit objects. Due to the fact all of the objects had absolute magnitudes of brighter than 16.5 , it is likely that the objects that were not found had eccentric orbits although it could be a brightness variation from the objects as well. Even with a variation of 1.5 in magnitude, the object would still have been within the limiting magnitude of the system. In addition, a study on the reacquisition of an eccentric orbit object when using ACO is discussed in the future observation section.

\section{October 2005}

Both the October 2005 and January 2006 runs were conducted over multiple nights with the first night being used for normal survey mode. The October 2005 run covered DOYs 278 - 280. In the first night, nine objects were detected. Each of objects was propagated to the next night (DOY 279) using an ACO. This was done as a test of the propagator to see if 24 hours was too 
long to recover objects. Thirteen objects were observed the second night, although only nine were observed the previous night. This posed a problem. How can one tell which objects were linked together? Without correlating the objects, each detection on the second night was combined with the detections from the first night, and the orbits were calculated and sent to the telescope for observations. It was noted which set of observations was more likely the true linkage based on the residual from the orbit fit to the observations; however, both sets of fields were observed.

After the January 2006 data collection, it was determined that the orbit with the lowest residual was usually the non-constrained-ecc orbit propagator solution and the priority was changed to reflect that. However, for this run, both predictions were sent to MODEST. The residuals are shown in Table 1 . In the table, the first column shows the detections for DOY 278 (objects 0001 - 0009). To explain the table better, an example is the first object, 0001, which on DOY 0001 had a residual from a circular orbit of 1.7. The predicted orbit was propagated to DOY 279. Observations from that field showed an object detected (0001). Combining those two observations yields a residual on the constrained-ecc orbit of 2.5 and on the non-constrained-ecc orbit of 1.5. Both orbit types were sent to MODEST for further inspection. Another example displays the results when two objects are found in the predicted field. On DOY 279, two objects were found at object 0003's predicted location (objects 0003 and 0004). Both objects were combined independently with object 0003 and predicted field locations were determined and sent to MODEST.

It was quickly apparent that the naming convention of these prediction files would need to be examined. A system in place now keeps track of the objects in order of observation so that when the run is finished the lineage of the detections would be preserved.

Because of the longer time arc ( 24 hours), an non-constrained-ecc orbit could be calculated with accuracy. Both the non-constrained and constrained orbits were tested and observed during this run. It was found though that the non-constrained-ecc orbit propagator solution proved to find a better orbit even when the object was in a near circular orbit. The third night of the October run found multiple objects in the predicted fields although only five of the objects seemed to be good matches with low residuals.

Post-observation correlation showed that three of the five objects were CTs and two objects were UCTs. All five objects had circular orbits.

\begin{tabular}{lllll}
\hline $\begin{array}{l}\text { DOY 278 } \\
\text { objects }\end{array}$ & $\begin{array}{l}\text { Residual DOY } \\
\text { circ }\end{array}$ & objects & $\begin{array}{l}\text { Residual Residual } \\
\text { circ }\end{array}$ & ecc \\
\hline 0001 & 1.69 & 0001 & 2.449 & $1.516^{*}$ \\
0002 & 2.261 & 0002 & 836.4 & err - div by 0 \\
0003 & 1.279 & 0003 & 41.11 & 29.79 \\
& & 0004 & 2.047 & $1.847^{*}$ \\
0004 & 0.9743 & 0005 & 4.354 & $1.782^{*}$ \\
& & 0006 & 26.48 & 25.99 \\
0005 & 1.02 & 0007 & 1.531 & $1.180^{*}$ \\
0006 & 0.9334 & 0008 & 4.489 & $3.196^{*}$ \\
& & 0009 & 285.5 & 274.8 \\
0007 & 0.8841 & 0010 & 3.28 & $2.111^{*}$ \\
0008 & 1.393 & 0011 & 2.331 & $1.855^{*}$ \\
& & 0012 & 162.6 & $162.3^{*}$ \\
0009 & 0.8802 & 0013 & 5.618 & $2.104^{*}$ \\
\hline
\end{tabular}

Table 1: Residuals for night 1 and night 2 of October 2005 data collection. The objects with the asterisk in the eccentric column depict the orbits that are still circular. The object numbers do not correlate with each other but are the order in which they were taken within the night.

January 2006

The final testing run was conducted in January 2006. During this run, the priority scheduling was used for the first time. The JSC staff determined the priority of the follow-up observations based on likelihood of future detection (shadow, predicted locations, etc.), the goodness of fit of the orbit, and the magnitude of the object. In these data shown in this paper, no correlation of the object to the catalogue is done while the observations are being conducted. From prior experiments, fainter objects are more often UCTs than CTs so it is assumed in this study that a fainter object is more likely to be a UCT rather then a CT. 


\begin{tabular}{lllllll}
\hline Object & Year DOY object \# & Number of Obs & ECC & Inc & MM & RAAN \\
\hline A & 20060310001 & 1 & 0 & 7.3951 & 0.995396 & 67.839 \\
A & 20060320102 & 2 & $1.29 \mathrm{E}-03$ & 7.3652 & 0.99759 & 67.583 \\
A & 20060320210 & 3 & $1.01 \mathrm{E}-03$ & 7.3686 & 0.997588 & 67.6212 \\
A & 20060321012 & 4 & $9.83 \mathrm{E}-04$ & 7.369 & 0.997587 & 67.6269 \\
A & TLE Prediction & & $8.42 \mathrm{E}-04$ & 7.35 & 0.9976 & 67.45 \\
& & & & & \\
B & 20060310011 & 1 & 0 & 5.0908 & 1.007234 & 79.6896 \\
B & 20060321103 & 2 & $7.71 \mathrm{E}-04$ & 5.0697 & 1.007703 & 78.992 \\
B & 20060320311 & 3 & $1.09 \mathrm{E}-03$ & 5.0686 & 1.007676 & 78.976 \\
B & 20060321113 & 4 & $9.86 \mathrm{E}-04$ & 5.0695 & 1.007663 & 78.9807 \\
B & TLE Prediction & & $7.36 \mathrm{E}-04$ & 5.05 & 1.0077 & 78.85 \\
& & & & & & \\
C & 20060310015 & 1 & 0 & 4.9342 & 1.001831 & 81.1507 \\
C & 20060321521 & 2 & $3.25 \mathrm{E}-03$ & 4.8742 & 0.99886 & 78.4943 \\
C & TLE Prediction & & $6.77 \mathrm{E}-04$ & 4.86 & 0.9988 & 78.42 \\
\hline
\end{tabular}

Table 2: Predicted Orbits for three objects observed in January 2006. As the number of observations of the object increases, the accuracy of the orbit increases.

To combat the issue of multiple objects found in the predicted field, each object is assumed to be the object in question until the residuals are examined. If the residual of the orbit, or goodness of fit, was low it was determined that it was the proper object. If the residual was high, the results were still sent to MODEST but the priority was set to a much larger number (priority of 1 is high priority and priority of 9 is low priority) to ensure that all viable observations were taken first. On the normal survey mode of data collection (DOY 030), 10 objects were detected. The following day (DOY 031) field locations based on an ACO were sent to MODEST. Objects were found in six of the 10 fields. It is possible that by not finding an object where the circular orbit predicts it to be that those objects were not in circular orbits. This information can be used in future observations by widening the search area for the object.

\section{$\underline{\text { Orbit Comparisons }}$}

A comparison of the orbits using the circular orbit assumption and the eccentric orbit propagator was conducted on three objects from the January 2006 observations. The results are shown in Table 2 and in Figures 2, 3, 4, and 5 for eccentricity (ecc), inclination (inc), mean motion (MM), and right ascension of ascending node (RAAN), respectively. In each of the figures, the final value is the predicted value from the two-line element set and is shown as an open symbol and is offset to ensure visibility.

Eccentricity, seen in Figure 2, has the largest increase in accuracy when more observations were added and the observed orbital arc was extended. In the first observation set, the eccentricity is basically set to zero, but as one adds more observations the eccentricity can be defined and refined. The inclination, shown in Figure 3, shows the least amount of change in accuracy by adding observations, although there is convergence toward the predicted value. Even though these objects are in circular orbits, adding observations lends an increase in accuracy for MM for all three objects as seen in Figure 4. The final orbital parameter, RAAN (shown in Figure 5), has less of a change in value when adding more observations. Like inclination, this means that the RAAN over a small time arc is likely accurate for dealing with nearly circular objects and both values may be unbiased by ACO for objects with moderate or high inclinations. 


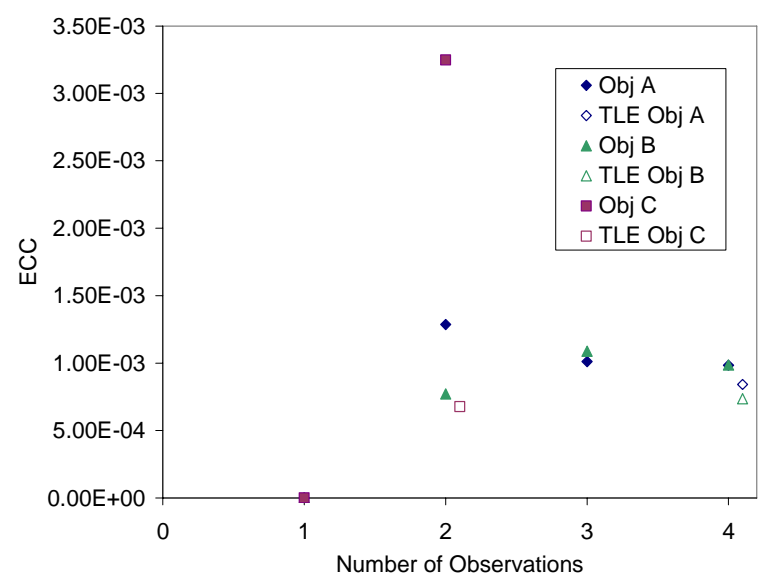

Fig. 2: Number of Observations versus the Eccentricity. Each filled point represents an observation. TLE value (open symbol) is offset from the last observation to ensure visibility.

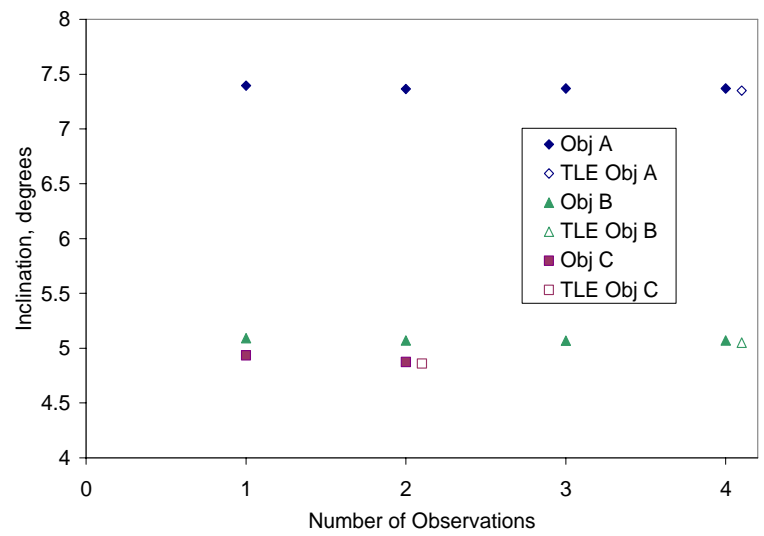

Fig. 3: Number of Observations versus Inclination, degrees. Same description of symbols as Figure 2.

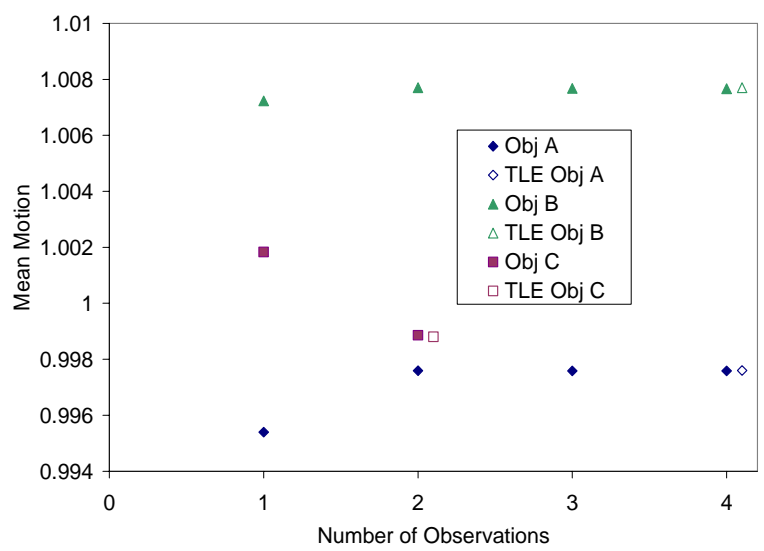

Fig. 4: Number of Observations versus Mean Motion, degrees. Same description of symbols as Figure 2.

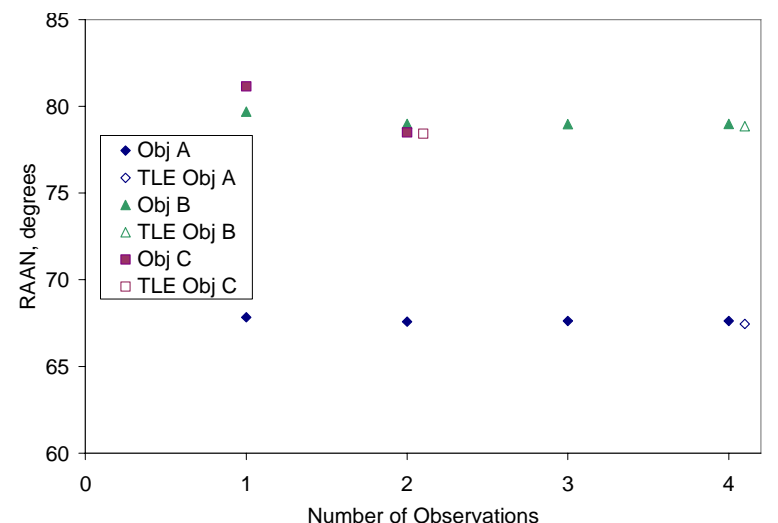

Fig. 5: Number of Observations versus RAAN, degrees. Same description of symbols as Figure 2.

\section{FUTURE OBSERVATIONS}

Due to the long-term goal of constructing a system which can detect and follow-up an object in any orbit at GEO, a system must be designed to obtain observations on objects which are not circular. A subset of CT eccentric orbit objects detected previously with MODEST during survey mode were propagated for times of 30 minutes through 8 hours past the last observation. This testing was designed to see if there was a systematic search pattern that could be followed after the survey observations were obtained to maximize the possibility of followon observations as well as the necessary handoff time to ensure observation. When the code could compute an eccentric solution on the short arc from the survey, both solutions are provided, however, for some of the objects the eccentric solution could not converge on an orbit and thus only the circular orbit prediction was calculated.

It was assumed that the telescope would be pointed at the RA and DEC at the specific time of the circular orbit prediction. Then, it was determined how long the object would have been in the FOV. Two objects are shown here as representative examples of the subset tested. Object A had an eccentricity of 0.18 and object $B$ had an eccentricity of 0.48 . Both objects were observed with MODEST during normal survey operations. Both figures show the circular orbit predicted (red line straight line), the eccentric orbit prediction (green line, or top curved line), the "true" orbit based on the two-line element (TLE) predictions (blue line, or the shortest 
line), and the actual observations (black line at the beginning of the UT).

Figure 6 shows the predicted RA and DEC for a given time for the circular, eccentric, and TLE orbits for an object (object A listed as obs) with eccentricity of 0.18 . The object would have been detected with the circular orbit assumption up to 1.35 hours from the first prediction and 2.5 hours after the first detections. This is shown in Figure 7. After 2.5 hours after the initial detection, the TLE prediction states the object would have gone into shadow, but prior to entering the shadow the likelihood of detecting this object would have been good.

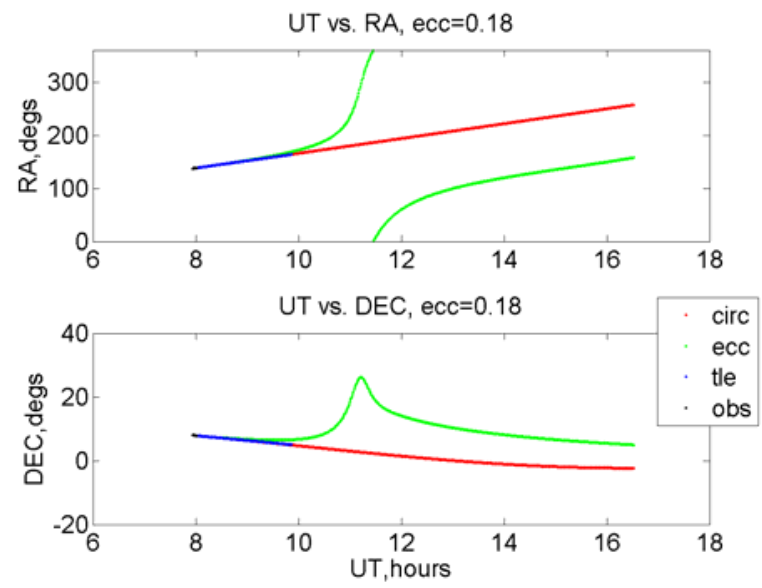

Fig. 6: Object A Universal Time versus RA and DEC.

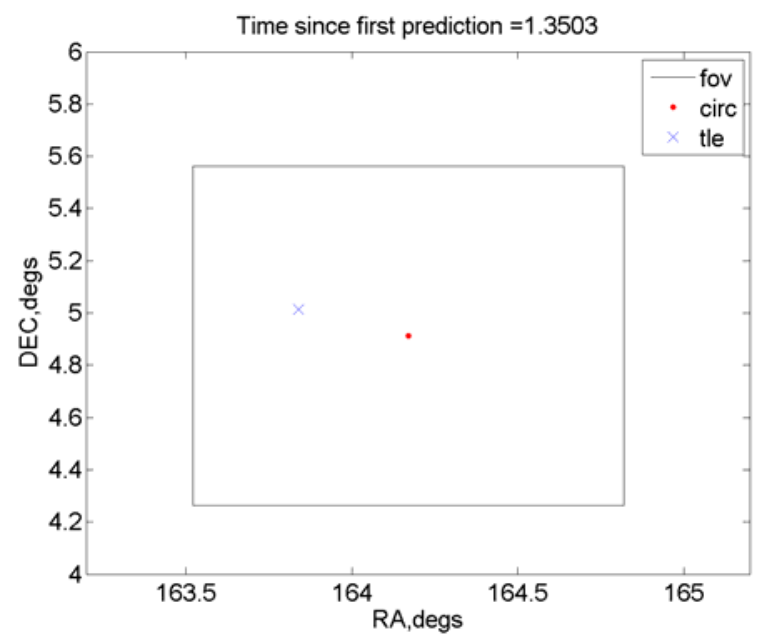

Fig. 7: Image of the FOV after 1.35 hours after the first prediction (2.5 hours after detection) showing the "true" position of the object is still in the FOV if a circular orbit is assumed.
Figure 8 shows object $B$ with the circular, eccentric, and TLE predicted orbits, and actual observations. Object B has an eccentricity of 0.48. If the telescope had been centered on this object at the location predicted by the circular orbit, we would have never seen the object in the 30 minute turn around assigned for this project as seen in Figure 9. In general, half of the eccentric objects tested would have been recovered as long as the observations took place within 30 minutes of the initial observation. This study showed that if survey observations are to continue as well as followup observations, two telescopes will be necessary so that the hand-off between the systems is as fast as possible and definitely within 30 minutes of the initial observations.

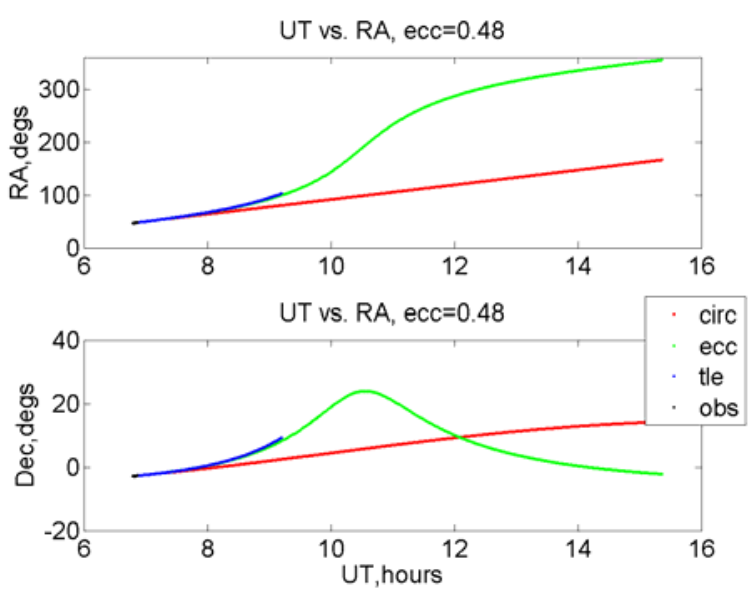

Fig. 8: Object B Universal Time versus RA and DEC.

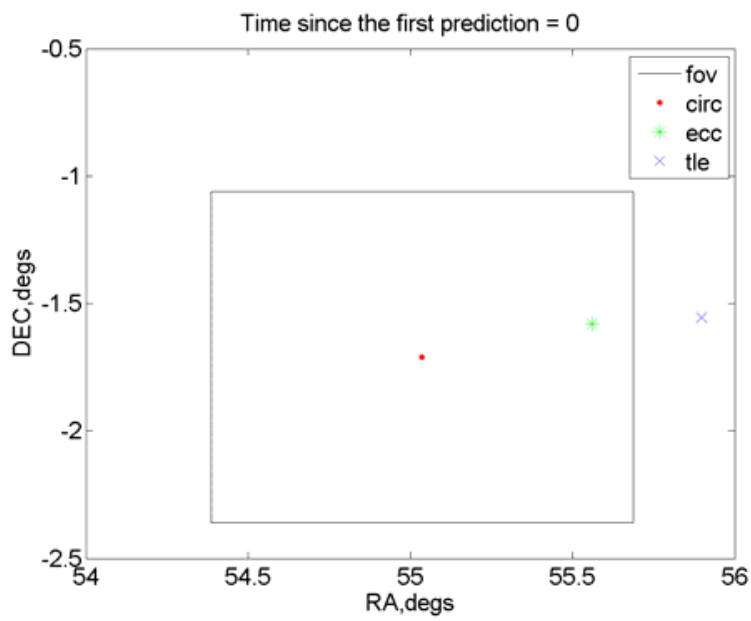

Fig. 9: Image of the FOV after 30 minutes showing the object would not be in the FOV if a circular orbit was assumed. 


\section{CONCLUSIONS}

The process of using follow-up (chase) observations to obtain a longer arc on a specific object was successful. By using a quick turn around method of orbit determination and propagation, one can obtain better orbital parameter accuracy. Real-time detection is possible with MODEST using fast reacquisition of an object.

It was surmised through these trial runs that in order to obtain eccentric orbit objects, the orbits for the follow-up observations would need to be updated as new observations of the object arrived. To obtain eccentric objects, one hour was too much time between the initial observations and the first follow-up measurement. Yet there exists a population of faint objects at GEO which are on eccentric orbits, as determined by the ESA $\mathrm{SDT}^{6}$, so determining eccentric orbits is not just an academic question.

In addition, the bookkeeping of what object was matched with what follow-up observation can be very confusing when observing multiple fields on multiple nights. This needs to be tracked carefully so that the validity of the orbital linkages are preserved.

The residuals increase when adding more observations and so depending on a specific value of the residual to determine the goodness of fit of an orbit is not appropriate. As more testing commences, the values will be tabulated and a priority will include the number of observations as well as the magnitude, location, and residual.

In the future, more correlation studies within a night and night to night will be conducted using these methods and building on the knowledge obtained. Future work on this project would be to continue to use MODEST in survey mode and have a second telescope dedicated to the followup observations. In this fashion, the knowledge of the statistical environment is gathered as well as orbital elements and photometric properties on specific objects.

\section{REFERENCES}

1. R. Musci, et al. Orbit Improvement for GEO objects using follow-up observations, Advances in Space Research, 34, 912, 2004.

2. R. Musci, et al. Orbit improvement for GTO objects using follow-up observations, Advance in Space Research, 35, 1236, 2005.

3. P. Seitzer, et al. A Survey for Space Debris in Geosynchronous Orbit in the Proceedings of AMOS 2001 Technical Conference, Maui, HI, 2001.

4. P Seitzer, et al. Results from the NASA/Michigan GEO Debris Survey, the Proceedings of AMOS 2004 Technical Conference Proceedings, Maui, HI, 2005.

5. R.R. Bate, D.D. Muller, and J.E. White. Fundamentals of Astrodynamics, Dover Publications, New York, NY, 1971.

6. T. Schildknecht, et al. Optical Observations of Space Debris in High-Altitude Orbits, in the Proceedings of the Fourth European Conference on Space Debris, Darmstadt, Germany, 2005. 